\title{
Sistema de Automatizado de Alerta Temprana ante Inundaciones SAATI y mesa de simulación
}

\author{
Diana Muñoz ${ }^{1}$ \\ Alonso Licona ${ }^{2}$ \\ Angela Morales ${ }^{3}$ \\ Fernando Zorto ${ }^{4}$
}

\section{RESUMEN}

Se diseñó y construyó una mesa de simulación de inundaciones provocadas por precipitación. La mesa consiste en una maqueta que representa un río, al cual se le añade agua almacenada en un tanque con el objetivo de que aumente el nivel de agua, simulando una inundación. Se desarrolló un sistema de alerta temprana que, a partir de datos de nivel de agua obtenidos mediante sensores ultrasónicos, emite alertas de inundación. Los sensores están colocados en puntos aleatorios equidistantes que envían los datos a una placa electrónica para ser procesados y posteriormente almacenados en una hoja de cálculo. La información es exportada nuevamente a la placa y los niveles de agua son comparados con los escenarios de riesgo establecidos. El código automáticamente evalúa la necesidad de emitir alerta verde, amarilla 0 roja, según sea el caso. El sistema tiene la capacidad de almacenar la información obtenida de manera periódica, generando una base de datos de nivel de rio en función del tiempo.

La mesa de simulación, además de servir como medio de verificación del código en laboratorio, servirá para la generación de nuevos prototipos. Con este sistema de alerta temprana se busca fortalecer los comités de emergencia a nivel local, brindado información en tiempo real que sirva para tomar decisiones de manera más efectiva.

Palabras clave: Alerta temprana, mesa de simulación, inundaciones.

\footnotetext{
${ }^{1}$ Estudiante de Ingeniería Civil, Facultad de Ingeniería, UNAH: diana.munoz@unah.edu.hn

${ }^{2}$ Estudiante de Ingeniería Eléctrica, Facultad de Ingeniería, UNAH. Co-investigador del proyecto SICOEEL: wmartinezl@unah.hn

${ }^{3}$ Estudiante de Ingeniería Civil, Facultad de Ingeniería, UNAH:angela.morales@unah.edu.hn

${ }^{4}$ Asesor Metodológico . Profesor de la Carrera de Ingeniería Mecanica-. Facultad de Ingeniería: fernando.zorto@unah.edu.hn
} 


\section{INTRODUCCIÓN}

Las inundaciones son una amenaza que está latente en la región centroamericana. Es usual que, en la época de lluvia, diversas zonas se vean afectadas por crecidas. Como medida de prevención de desastres, se adoptan los sistemas de alerta temprana ante inundaciones, los cuales, en su mayoría son manuales. Esto conlleva una serie de errores en la toma de datos, así como exposición a peligros de las personas encargadas de la medición, al tener que estar cerca del rio.

Honduras ha sido afectada por numerosos eventos desastrosos de origen hidrometeorológico. Entre estos eventos destacan: la Tormenta Tropical No. 13 (1949), el Huracán FIFI (1974) y el Huracán Mitch (1998). La Fig. 1 muestra ejemplos de los daños ocasionados por los huracanes.

Figura 1. El huracán Mitch causo severas inundaciones en gran porcentaje del territorio hondureño.

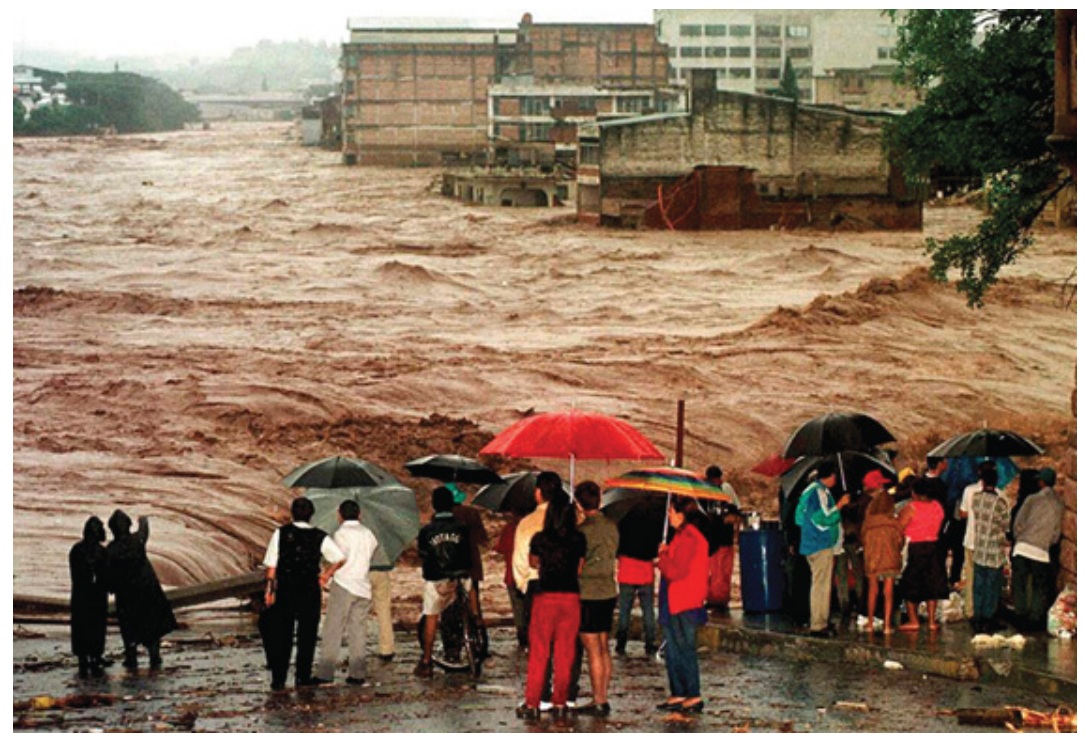

Fuente: Diario La Tribuna. 28.oct.2016 http://www.latribuna.hn/2016/10/28/18-anos-del-mitch-honduras -avanza-ruta-del-desarrollo/

Según datos del Programa de las Naciones Unidas para el desarrollo en el 2011, se estima que las pérdidas financieras en Honduras durante los últimos treinta años debido a los desastres son de $\$ 4.7$ billones, representando aproximadamente el $50 \%$ de las pérdidas de Centroamérica (PNUD, 2011). Además, a pesar de los impactos 
recurrentes de las tormentas tropicales y huracanes, en el país todavía existe una capacidad de adaptación muy baja a nivel nacional. Una gran porción de la población del país permanece en riesgo severo de eventos hidrometeorológicos y eventos climáticos extremos asociados, tales como inundaciones, sequías, y deslizamientos. En Honduras se han instalado sistemas de alerta temprana, con ayuda que brindan los países cooperantes. Un ejemplo es la obra de mitigación ubicada en El Berrinche, Tegucigalpa, la cual fue donada por la Agencia de Cooperación Internacional del Japón (JICA). La obra cuenta con un sistema de alerta temprana ante deslizamientos que consta de una serie de canales y pozos que dirigen el agua subterránea al rio Choluteca, evitando así que el suelo se sature.

\section{Figura 2. Obra de mitigación en Cerro el Berrinche.}

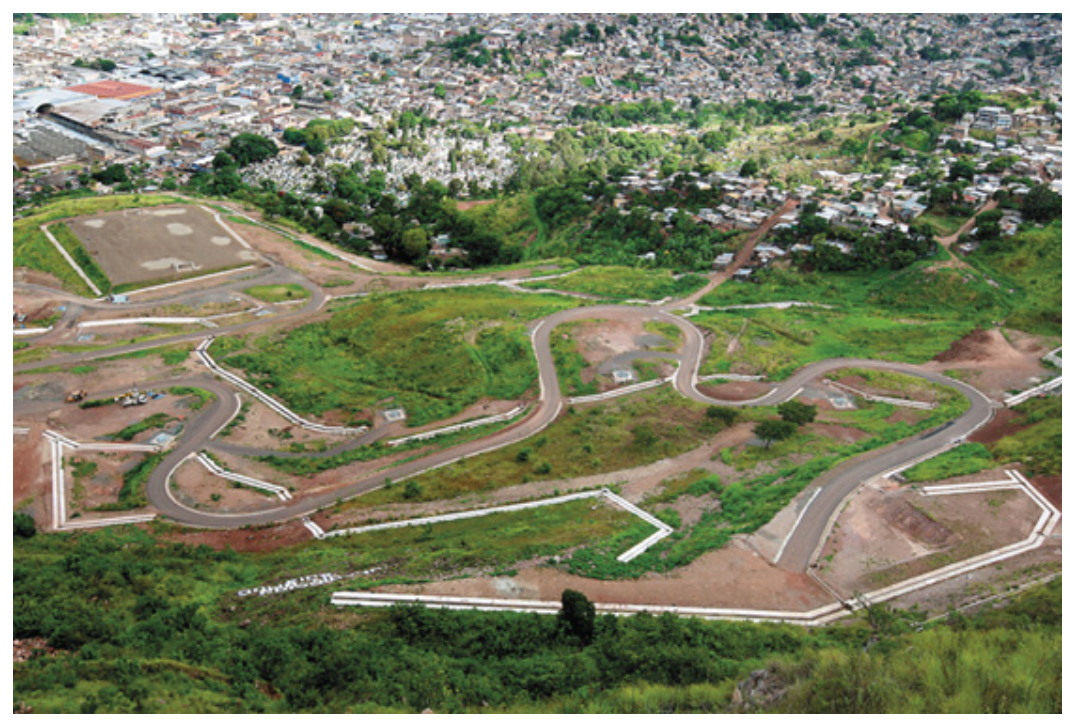

Fuente: Diario El Heraldo 7.abr.2014 https://www.elheraldo.hn/metro/587487-213/m\%C3\%A1s-de $-81-a \% C 3 \% B 10 s-d e-p \% C 3 \% A 1$ nico-finalizan-en-el-berrinche

En el Distrito Central se han desarrollado diversos proyectos para alertar a la población ante una posible amenaza de inundaciones, entre ellos se encuentra el proyecto "Fortaleciendo la Operatividad de los Sistemas de Alerta y Acción Temprana (SAAT) para el Distrito Central". El programa es financiado por el Fondo de Adaptación al Cambio Climático y ejecutado a través de la Secretaría de MiAmbiente y GOAL, y busca salvar vidas de los habitantes en las zonas de riesgo, debido a que Tegucigalpa es una de las ciudades más vulnerables frente a fenómenos hidrometeorológicos (Diario LaTribuna, 2016) 
Figura 3. Sistema de alerta temprana ante inundaciones, donde se observan los distintos niveles de alerta

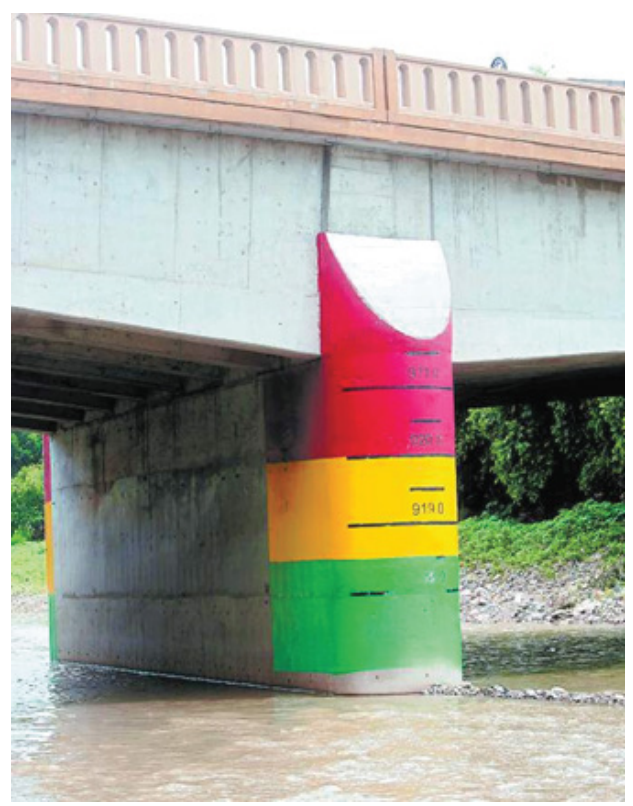

Fuente: Diario La Tribuna 16.jun.2015 https://www.elheraldo.hn/metro/850236-213/unos-52-barrios-ycolonias-bajo-amenaza-de-inundaci\%C3\%B3n

En general, un sistema de alerta temprana lo componen tres elementos básicos:

1) Sensores de lluvia y telemetría.

2) Umbrales críticos de lluvia definidos por modelos físicos o estadísticos.

3) Medios para la comunicación de las alertas a las autoridades y comunidad.

La implementación y desarrollo (automatización) de un sistema de alerta temprana ha surgido de la necesidad de mejorar y prever las alteraciones naturales, ya que esto conlleva a múltiples crisis, tanto económicas como pérdidas humanas. Se trata de anticiparse a estos hechos mediante el uso de estudios y herramientas adaptados a las zonas de alto riesgos a inundaciones, deslizamientos y demás factores naturales que puedan ocasionar una catástrofe.

Existen dos métodos de estimación de precipitaciones: estimación de precipitación cuantitativa (QPE por sus siglas en inglés) y pronóstico de precipitación cuantitativa (QPF por sus siglas en inglés). Con base en estos dos métodos, "existen estaciones que miden la lluvia cuantitativamente, como los pluviógrafos, e instrumentos que pueden estimar y pronosticar espacial y temporalmente la lluvia, como los radares. 
Por otro lado, "los radares transmiten pulsos de energía de microondas que son reflejados por las gotas de lluvia y recibidos por las antenas. La señal de reflectividad se relaciona con la intensidad de la lluvia" (Aristizábal, et. al. 2015).

Algunos sistemas han sido desarrollados basados similarmente en umbrales de lluvia críticos en diferentes lugares como ser:

En Japón se desarrolló un sistema para la alerta y evacuación basado en el proceso de infiltración y el flujo superficial. Se definieron cantidades de lluvia estándares determinadas como acumulaciones críticas de lluvia dentro de ciertos periodos de concentración, combinadas con modelos de estabilidad estáticos. Para mejorar la precisión de las predicciones, se combinaron estaciones de lluvia en tierra con mediciones de radares y telemetría en tiempo real (Guzzetti, et. al, 2015).

En Río de Janeiro, GeoRio (Oficina de Ingeniería Geotécnica de Río de Janeiro) diseñó en 1996 un sistema conformado por una red de 30 estaciones de lluvia en tiempo real y radares meteorológicos, denominado Sistema de Alerta Río. Este sistema genera pronósticos y alertas para movimientos en masa e inundaciones rápidas a las agencias gubernamentales y la comunidad durante tormentas intensas (Aristizábal, et. al. 2015). El objetivo de esta investigación es brindar un sistema capaz de emitir alertas ante inundaciones. SAATI se compone de dos elementos importantes: una mesa didáctica de simulación de inundaciones y un sistema de monitoreo y alarma automática. Este sistema tiene como función principal la detección temprana de inundaciones provocadas por precipitación, además de almacenar datos en tiempo real para ser usados posteriormente en estudios e investigaciones que requieran datos de niveles de río y caudal. Con la mesa de simulación se pretende mostrar a los estudiantes cómo se comporta una crecida, además de ser una herramienta para desarrollar posteriores investigaciones.

\section{MATERIALES Y EQUIPO}

\section{Mesa de simulaciones}

El interior de la mesa contiene la sección de un río. También se pueden observar el canal y las curvas de nivel. La mesa está conformada por una estructura metálica que le brinda soporte. El contenedor tiene una dimensión de 215 × $950 \mathrm{~cm} 2$ con una altura de $30 \mathrm{~cm}$. Las dimensiones de la mesa y las vistas del diseño se pueden observar en 
las Figs. 4, 5 y 6 . En la fig. 7 se puede observar el montaje final de la mesa.

La forma del río fue creada con láminas de poliestireno e impermeabilizado con masiIla a prueba de agua. Fue necesario colocar un plástico previo a la colocación del poliestireno para evitar el óxido y posibles fugas, además de sellar las uniones con silicona para fontanería.

El agua se distribuye en el río por medio de tubería PVC de $1 / 2$ pulgada de diámetro que está conectada a un tanque de 250 litros. Debido a que el agua recircula, el tanque solamente es vaciado para su limpieza y mantenimiento. El agua es impulsada por una bomba de $0.5 \mathrm{Hp}$ y $110 \mathrm{~V}$ y se puede regular el flujo de entrada y salida a la mesa mediante una serie de válvulas instaladas.

Figura 4. Esquema de mesa vista lateral (izquierda) y frontal (derecha)
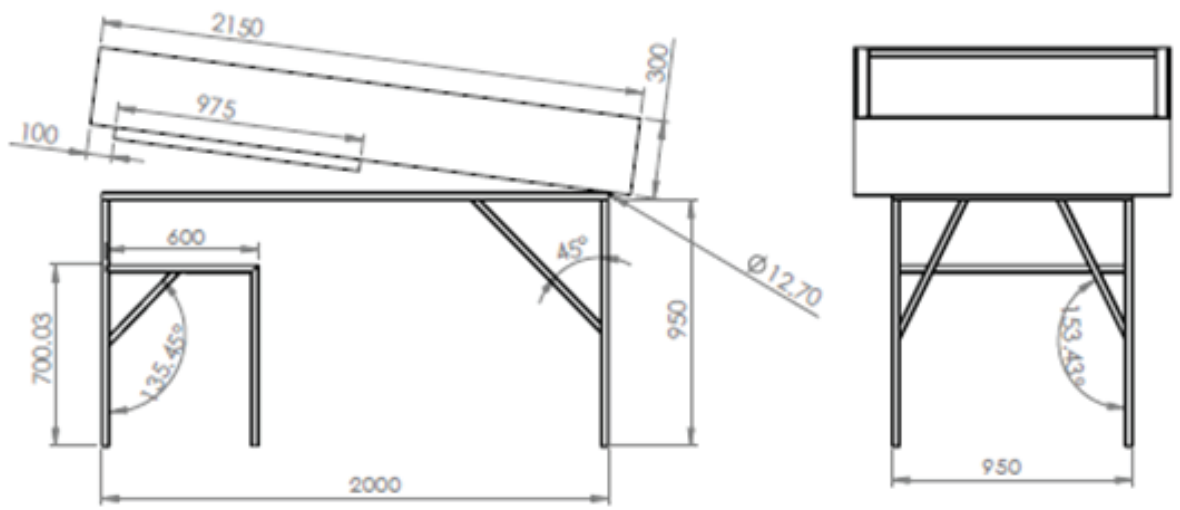

Figura 5. Esquema de mesa vista inferior

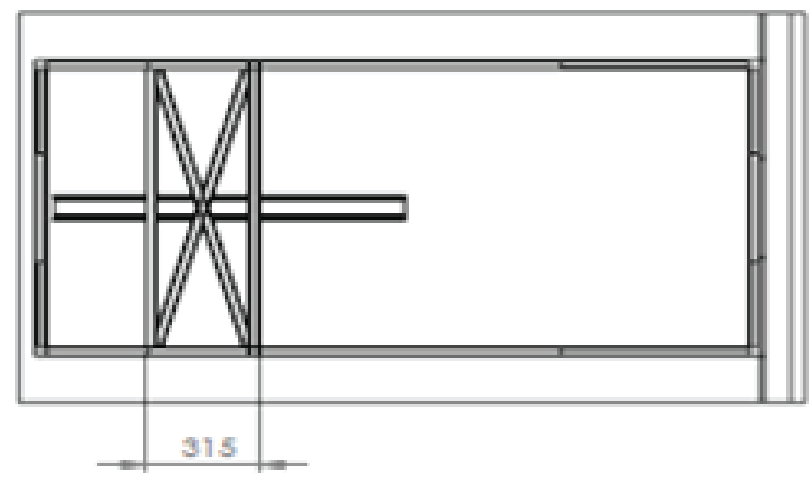


Figura 6. Dibujo isométrico de la mesa.

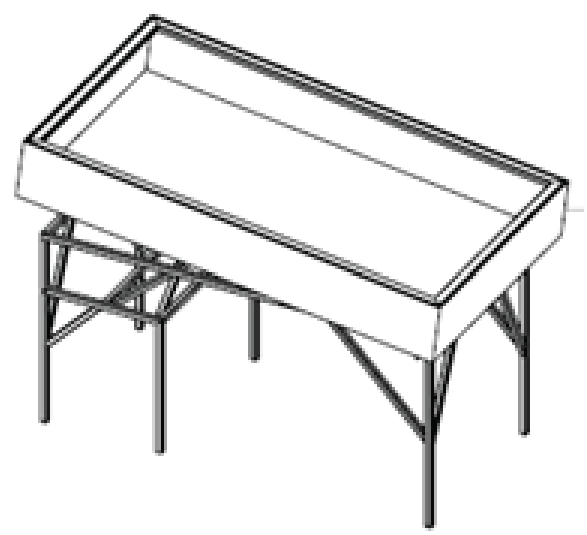

Figura 7. Montaje final de la mesa de simulaciones y componentes

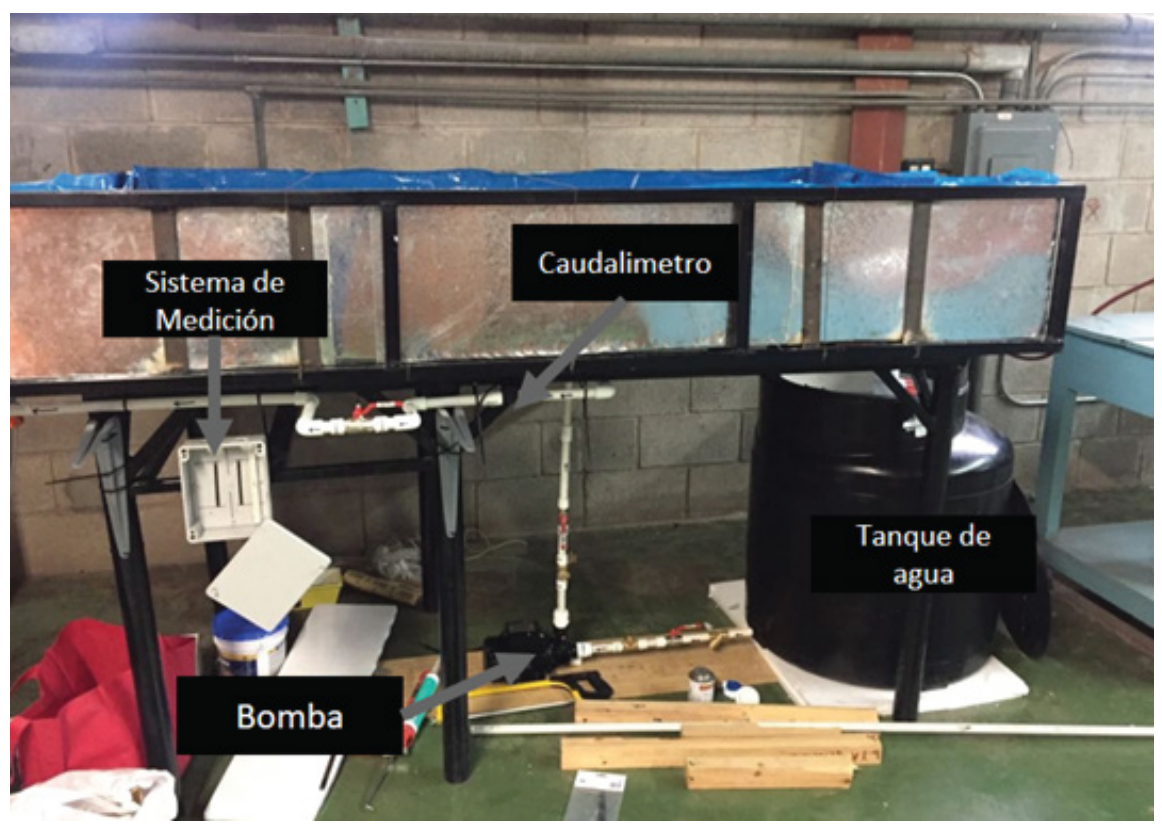

Hardware del sistema

Placa electrónica: La placa electrónica usada es la Intel Edison. El "Intel Edison Compute Module" es el dispositivo que recibe, interpreta y actúa con base en la adquisición de datos, es decir, se encarga de la parte lógica. Las especificaciones se muestran en la Tabla 1. 
Tabla 1. Especificaciones placa Intel Edison Compute Module (Intel, 2016).

\begin{tabular}{|l|l|l|l|l|}
\hline Dimensiones & Memoria & Conectividad & Puertos & Otros \\
\hline $35.5 \times 25 \times 3.9 \mathrm{~mm}$ & $\begin{array}{l}\text { 1GB de RAM, } \\
\text { GGB de } \\
\text { almacenamiento }\end{array}$ & WiFi y bluetooth & 2 puertos UART & $\begin{array}{l}\text { posibilidad de } \\
\text { conectar una } \\
\text { memoria SD }\end{array}$ \\
\hline
\end{tabular}

Los recursos y conectividad de la placa Intel Edison permiten una gran facilidad para que un proyecto en desarrollo pueda ir mutando a medida se le realicen mejoras. Por ejemplo, en esta etapa se usó conexión serial usando puertos UART-USB (conexión física mediante cables) pero se podría hacer a su vez el internet de las cosas (IoT) usando conectividad WiFi; si a esto se le suma su tamaño, también puede perfectamente ser portable. Cabe aclarar que el módulo Edison se adaptó a un kit o placa extensora (Intel, 2017) solamente para facilidad de maniobra.

Sensores: La obtención del nivel de río se realiza mediante sensores ultrasónicos de distancia modelo HC-SR04, instalados en tres puntos a lo largo de la mesa de simulación (ver Fig.8). Los sensores miden la altura del agua y determinan en qué posición se está generando la crecida. La distancia se mide en centímetros, pero es adaptable a cualquier unidad. El caudal se mide a través de un caudalímetro modelo YF-S201 (conexión de $1 \frac{1}{2}$ pulgada) que va conectado a la tubería.

Figura 8. Ubicación de los tres sensores ultrasónicos a la mesa de simulación

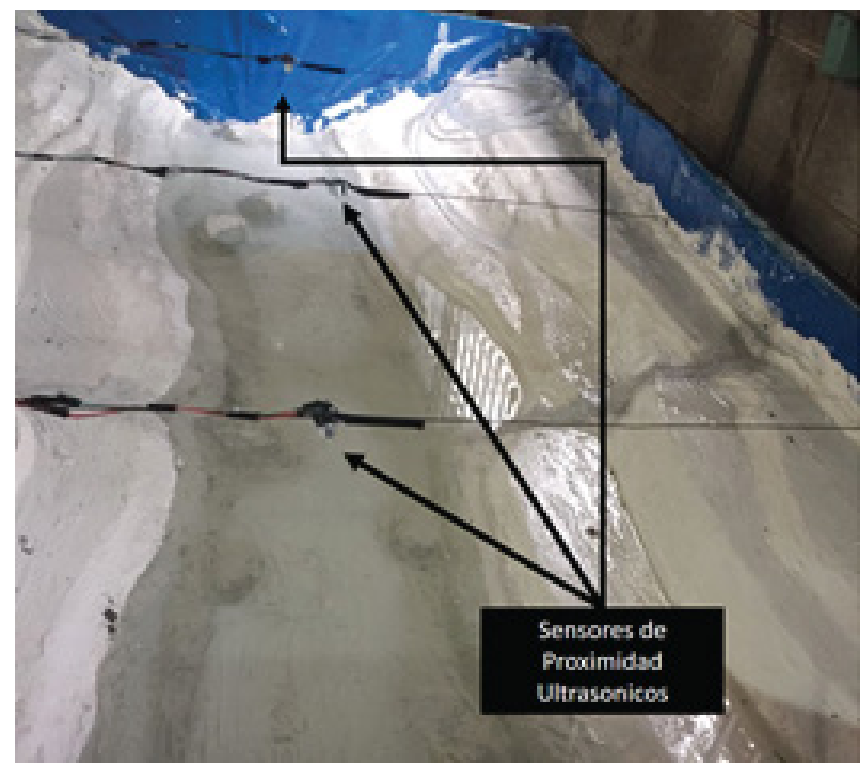


Ordenador: Se puede utilizar cualquier computadora que cuente con los drivers instalados que se requieren para ejecutar todo lo competente al software de SAATI.

\section{METODOLOGÍA}

El sistema funciona a través de un conjunto de sensores que recolectan la información de nivel de río y caudal. La información pasa a través de un controlador (la placa Edison) donde los datos son procesados y, en caso de ser necesario, se emite la alerta. Toda la información que pasa por el controlador es enviada a un software de análisis matemático para exportarla a una hoja de cálculo y a su vez, estas hojas de cálculo son enviadas a una base de datos. En la fig. 9 se puede ver el diagrama de funcionamiento del sistema de alerta temprana.

\section{Figura 9. Diagrama del funcionamiento de SAATI y su posible aplicación.}

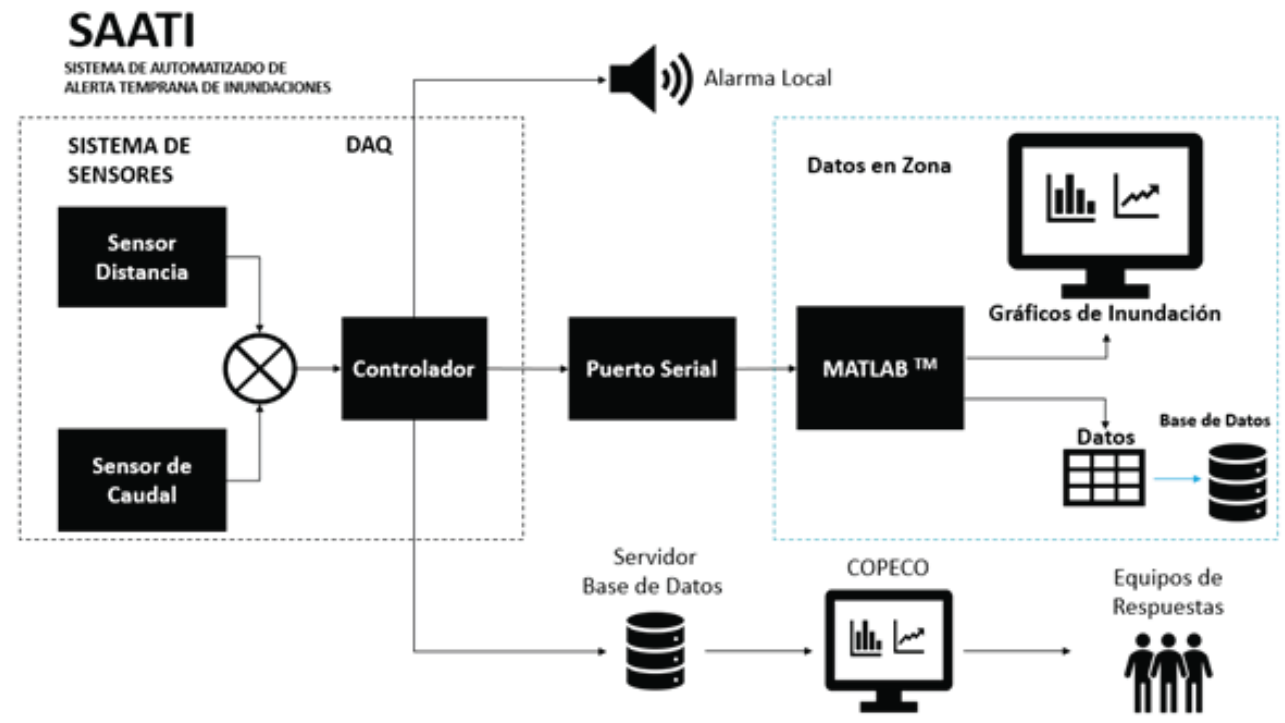

El código de programación fue escrito en el software Entorno de Desarrollo Integrado (IDE) de Arduino. IDE - Arduino es una aplicación multiplataforma escrita en el lenguaje de programación Java. Posteriormente, el código fue compilado al microprocesador de la placa electrónica. Dicho código tiene el fin de estructurar e indicar las funciones lógicas que deberá realizar la placa. Así mismo, se desarrolló un aplicativo en Matlab para obtener los datos de los sensores y luego exportarlos a un libro de 
Excel. El código fue pensado para que pueda detectar las crecidas en tres alertas, (verde, amarilla y roja), en función de la altura del río, además de leer el caudal entrante al sistema.

La Fig. 10. muestra la conexión real que se hizo en la placa de prueba (protoboard) para lograr la alimentación de los sensores y la transferencia de datos hacia la placa. Las líneas amarillas representan la conexión de transferencia de datos, las rojas representan la alimentación de $5 \mathrm{~V}$, y las negras representan la conexión al GND (Ground) que es la toma de tierra, OV.

Figura 10. Montaje de placa y sensores.

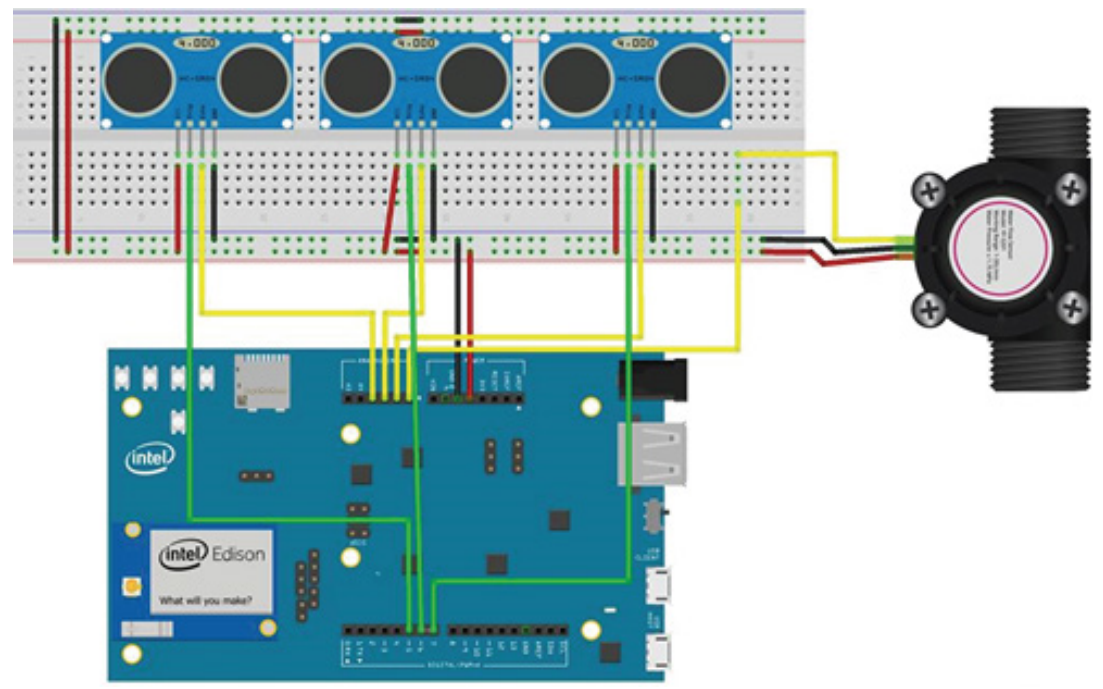

Los umbrales son los nivele máximos de riesgo que se pueden asumir. En un río, existe una altura máxima donde se considera que todo está dentro de lo normal. Al superar esa altura, se empiezan a tener los diferentes niveles de alerta verde, amarilla o roja que dependen del incremento en el nivel del río. Para determinar estos umbrales, es necesario realizar estudios que permiten modelar el río y definir los diferentes escenarios.

Para la mesa de simulación, los umbrales se definieron de manera aleatoria dado que solo se necesitaba que el dispositivo diferenciara los intervalos y emitiera las diferentes alarmas. En campo, se le deben ingresar al programa las distancias reales. 


\section{RESULTADOS Y DISCUSIÓN}

Las Fig. 11, 12, y 13 corresponden a las gráficas generadas por los sensores 1, 2 y 3 , respectivamente. La gráfica de la Fig. 8 muestra una tendencia diferente a las gráficas de los otros sensores, debido a que se hizo una desconexión temporal durante la realización de la prueba aquí detallada.

Se puede observar en las Fig. 12 y 13 la tendencia de la crecida del canal. Se inicia con una altura base de 4 centímetros aproximadamente, llegando a una crecida máxima diferente para cada sensor, dado que la mesa puede inclinarse a diferentes ángulos para simular la pendiente de un río. Por tanto, es de esperarse que el sensor que presente mayor crecida sea el número 3 . El sistema de alerta temprana activa sus alarmas dependiendo de los umbrales que se le ingresen; puede que una crecida no represente una gran amenaza, pero eso se determina mediante los umbrales.

Es posible obtener el caudal a partir del área y las alturas de crecida, haciendo uso de la Ecuación 1. A partir de ello, se puede generar el hidrograma de crecida, el cual compara caudal versus tiempo.

Ecuación 1.

$$
\mathrm{Q}=\text { área } \frac{1}{\mathrm{n}} \mathrm{R}^{\wedge 2 / 3} \sqrt{\mathrm{S}}
$$

Donde:

$\mathrm{Q}=$ caudal

Caudal $=$ área * velocidad

Área= anchura media * altura media

$\mathrm{n}=$ parámetro de rugosidad de Manning

$\mathrm{R}=$ radio hidráulico

$\mathrm{S}=$ pendiente $(\mathrm{m} / \mathrm{m})$

Figura 11. Nivel de rio vs. tiempo (sensor 1).

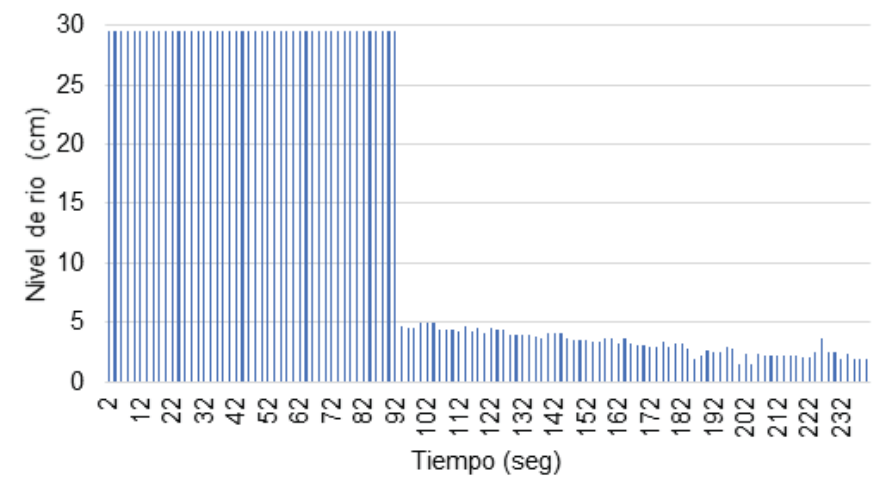


Figura 12. Nivel de rio vs. tiempo (sensor 2).

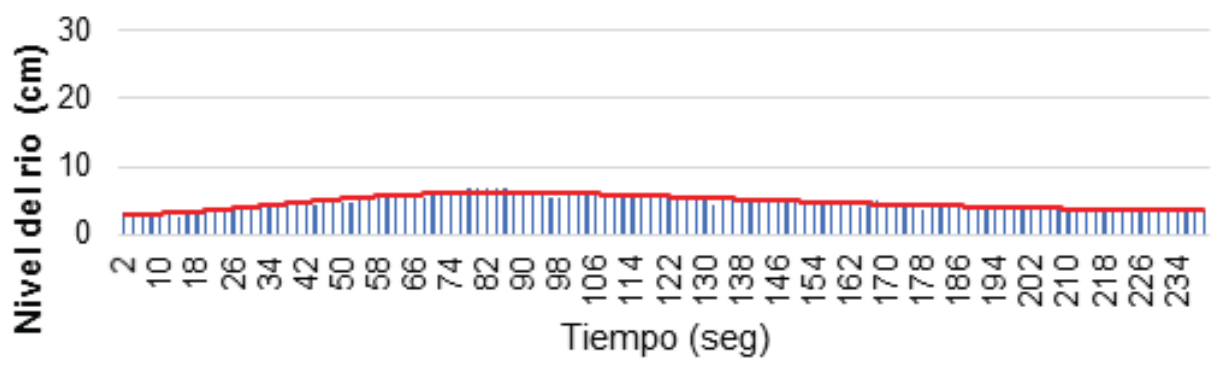

Figura 13. Nivel de rio vs. tiempo (sensor 3).

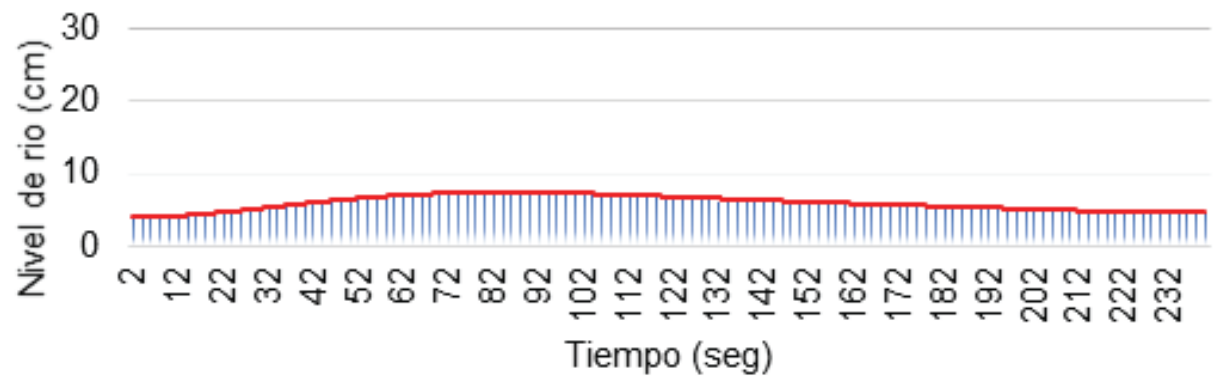

Figura 14. Comparación sensores 2 y 3.

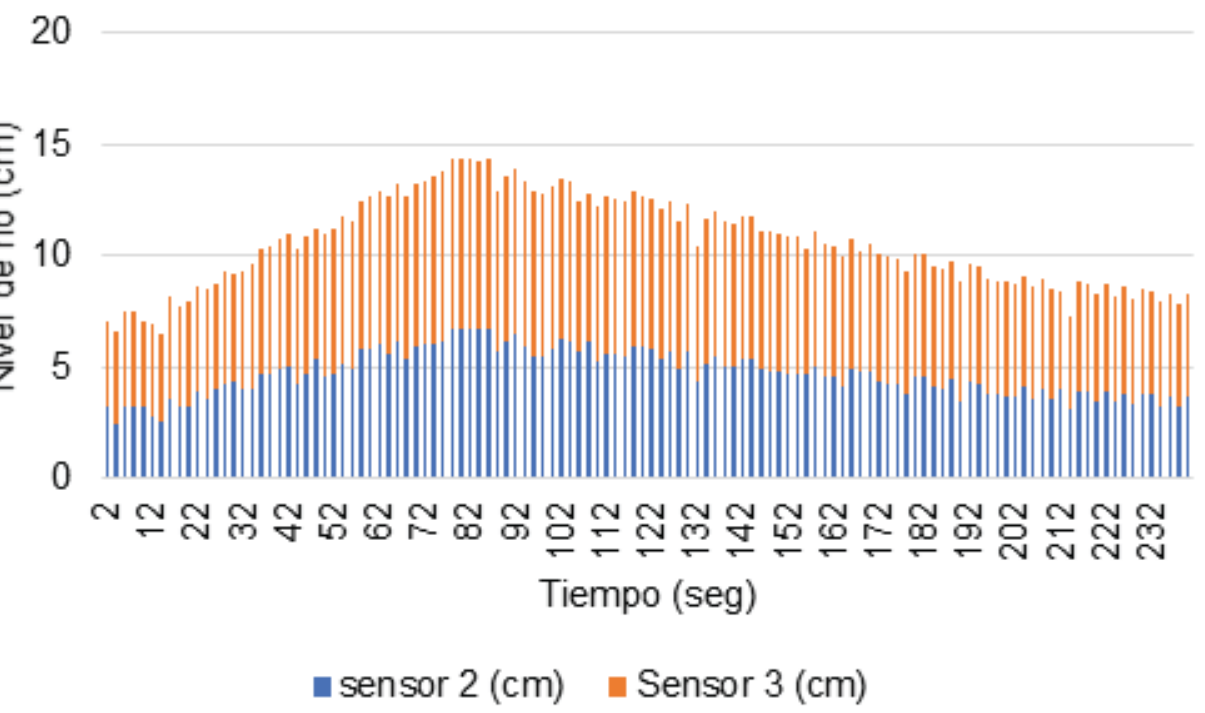




\section{CONCLUSIONES}

1. Se construyó una mesa de simulación de inundaciones, la cual cuenta con el perfil de un rio genérico. La mesa es monitoreada por una serie de sensores, logrando obtener la información de niveles de río y caudal, necesaria para el funcionamiento del Sistema de Alerta Temprana.

2. El algoritmo desarrollado es capaz de emitir los niveles de alerta, interpreta y almacena los datos obtenidos de los elementos de la mesa de simulación.

3. Las simulaciones realizadas se llevaron a cabo con éxito, así como el almacenamiento de los datos en una hoja de cálculo de manera continua.

\section{RECOMENDACIONES}

1. El desarrollo del dispositivo debe seguir avanzando en torno a la comunicación de la alerta para que ésta se realice de manera eficiente y de acuerdo a las condiciones imperantes en ese momento.

2. El dispositivo debe ser calibrado y probado en campo para asegurar su correcto funcionamiento a la intemperie.

3. Se debe realizar un manual de uso de los dispositivos para que puedan ser usados en futuras investigaciones y en clases.

4. Se puede buscar una reestructuración, ya sea del software y manejo de datos, para facilitar su uso.

\section{AGRADECIMIENTOS}

Se agradece a la Universidad Nacional Autónoma de Honduras por todos los recursos proporcionados para la realización de la investigación.

Al Laboratorio de Ingeniería Civil por brindar el espacio donde se desarrollaron los ensayos

Al Departamento de Ingeniería Mecánica por el respaldo académico y por facilitar sus laboratorios para la culminación de la mesa de simulaciones. 


\section{REFERENCIAS BIBLIOGRÁFICAS}

Aristizábal, E., Gamboa, M. F., Leoz, F. J. (2010). Sistema de alerta temprana por movimientos en masa inducidos por lluviapara el valle de aburrá, colombia. Revista EIA, 7 (13), 155- 169. doi: http://hdl.handle.net/11190/189

Guzzetti, F., Peruccacci, S., \& Rossi, M. (2005). Definition of critical threshold for different scenarios. RISK-Advance Weather forecast system to advice on Risk Events and management.

Intel. (2016). Intel Edison Compute Module. Obtenido de https://www.intel.com/content/dam/support/us/en/documents/edison/sb/edison-module_HG_331189.pdf

Intel. (2017). Intel Edison Kit for Arduino. Obtenido de https://www.intel.com/content/dam/support/us/en/documents/edison/sb/edison-arduino-hardware-guide.pdf

International Early Warning Programme IEWP (2005). Dedicated to Reducing Disasters through Effective People-Centered Early Warning Systems, World Conference on Disaster Reduction, Kobe, Hyogo, Japan.

Orrego, J. C. (2013). Construyendo Resiliencia en Honduras: Transformación de las Capacidades en Honduras para Lograr Mayor Resiliencia frente a los Desastres. Honduras.

Programa de las Naciones Unidas para el Desarrollo PNUD. (2011). Enfrentando riesgos climáticos en recursos hídricos en Honduras: Incrementando resiliencia y disminuyendo vulnerabilidades en áreas urbanas probres. Tegucigalpa.

La Tribuna. (2016). Instalan sistema de alerta temprana en la capital. Obtenido de http://www.latribuna.hn/2016/05/04/instalan-sistema-alerta-temprana-la-capital/ 